\title{
Fuerza Laboral que Envejece, ¿Qué Hacer ante esta Tendencia?
} AGEING WORKFORCE: WHAT DO TO THIS TREND?

Alicia Hermosilla-Ávila', Tatiana Paravic-Klijn², Sandra Valenzuela-Suazo²

1. Programa de Doctorado en Enfermería Universidad de Concepción. Departamento de Enfermería. Universidad del Bío Bío, Chillán, Chile.

2. Facultad de Enfermería. Universidad de Concepción, Concepción, Chile..

\section{RESUMEN}

Objetivo: Conocer las diversas implicancias del envejecimiento en la fuerza laboral activa, sus consecuencias y las posibles estrategias ante esta tendencia desde el punto de vista de la salud y la enfermería del trabajo. Método: Se realizó una revisión narrativa consultando diversas bases de datos y textos relacionadas con la temática. Desarrollo: Se exponen diferentes enfoques del envejecimiento de las personas trabajadoras, considerando su desarrollo humano como un fenómeno multidimensional, que involucra una serie de consecuencias que afectan directamente al desempeño laboral actual. De este modo, se analizan los desafíos enmarcados en las necesidades de salud convocadas por los muchos problemas del adulto trabajador envejecido y se proyecta una serie de estrategias ante esta tendencia. Conclusiones: El envejecimiento de la fuerza laboral y los años de vida socialmente productivos se ven influenciados por varios factores, en donde la condición de salud delimita la calidad y prolongación del período de trabajo activo, por lo cual, el rol de enfermería es preponderante en la protección de la salud y la prevención de accidentes y enfermedades profesionales, guiando por medio de su rol promotor a diversas entidades, fomentando la intersectorialidad con involucramiento de la familia, la comunidad, las empresas y entes afines al trabajador, con el objetivo de propiciar lineamientos y oportunidades para un envejecimiento activo y saludable.

(Hermosilla-Ávila A, Paravic-Klijn T, Valenzuela-Suazo S, 2015. Fuerza Laboral que Envejece, ¿Qué Hacer ante esta Tendencia?. Cienc Trab. Sep-Dic; 17 [54]: 166-170).

Palabras claves: ENVEJECIMIENTO, TRABAJADORES, SALUD LABORAL, ENFERMERÍA DEL TRABAJO.

\section{ABSTRACT}

Objective: To know the various implications of aging on the active labor force, its consequences and possible strategies to this trend from the point of view of health and nursing work. Method: Narrative review made by consulting various databases and texts related to the topic. Development: In the study are exposed different approaches the aging of working people, given its human development as a multidimensional phenomenon that involves a series of consequences that directly affect the actual job performance. Thus, are analyzed the challenges framed in health needs convened by the many problems of the aged adult worker and a number of strategies projected with this trend. Conclusions: The aging of the workforce and socially productive years of life are influenced by several factors, where the health condition defines the quality and extension of the period of active work, so the nursing role is predominant in the health protection and prevention of occupational accidents and diseases, through its leading role in promoting in various entities, promoting intersectoral involvement with the family, the community, companies and entities related to the worker, with the aim of promoting guidelines and opportunities for an active and healthy aging.

Keywords: AGING, WORKERS, OCCUPATIONAL HEALTH, OCCUPATIONAL NURSING.

\section{INTRODUCCIÓN}

En la actualidad es evidente que las personas están viviendo más años que antes y tenemos un gran número de personas mayores envejecidas conviviendo en cada una de las familias, entendiendo este proceso como irreversible, multidimensional y que ocurre biológicamente a lo largo de toda la vida, incluso marcado por designios sociales, políticos, culturales y jurídicos ${ }^{1}$, y que invo-

Correspondencia / Correspondence:

Tatiana Paravic-Klijn

Facultad de Enfermeria. Universidad de Concepción

Roosevelt esquina Janequeo s/n. Concepción, Chile

Teléfono: (56) (41)2204449-2207065

e-mail: tparavic@udec.cl

Recibido: 30 de Septiembre de 2015 / Aceptado: 25 de Octubre de 2015 lucra deterioros progresivos, notoriamente fisiológicos y funcionales, con la adquisición de patologías crónicas degenerativas como las enfermedades cardiovasculares, demenciales, osteoarticulares, sensoriales y cánceres. ${ }^{2}$

Tan sólo para el año 2020 las personas sobre 64 años habrán aumentado en un $31,9 \% \%^{3}$, proyectando ya para el 2025 un adulto mayor por cada menor de 15 años y para el 2050 el triple de población anciana respecto a la actualidad mundial. ${ }^{4}$ Específicamente en Latinoamérica y el Caribe para el 2025 podrían alcanzar hasta el 25\% los adultos mayores de 64 años, y en Europa para el 2050 incrementarse en un 77\%, presentando cifras similares Estados Unidos y Asia. ${ }^{5-7}$ Hoy Chile ya cuenta con 2,6 millones de chilenos con edad sobre 60 años, igualándose para el 2025 el número de niños con el número de adultos mayores. ${ }^{1}$

Con una tendencia similar, la población de adultos mayores de 60 años activos laboralmente, en las naciones desarrolladas, para el 2050 aumentará de un $20 \%$ a un 33\%, con una proyección de 58 
millones de trabajadores sobre 64 años sólo en la Unión Europea, es decir, aumentará la edad de la fuerza de trabajo. ${ }^{8,9}$ Fenómeno debido principalmente a los cambios legislativos sobre las condiciones de acceso a las pensiones de jubilación y los cambios generacionales en las mujeres, resultando un crecimiento cada vez más rápido de la población jubilada y de la población activa envejecida. ${ }^{5,10}$

El presente artículo expone las diversas implicancias del envejecimiento en la fuerza laboral activa, sus consecuencias y las posibles estrategias ante esta tendencia desde el punto de vista de la salud y la enfermería del trabajo, por medio del análisis de una revisión narrativa efectuada mediante la consulta a diversas bases de datos como Web of Science, Scielo, Medline Pubmed, entre otras, desde el año 1989 hasta el 2014, junto a libros relacionados con la temática.

\section{DESARROLLO}

El envejecimiento ha sido explicado desde distintas teorías que, en su mayoría, conciernen a la vinculación del genoma y el medio ambiente como causantes, dentro de las cuales destacan: la teoría de regulación genética, en que el envejecimiento consiste en un desequilibrio entre los factores genéticos que mantienen la fase de reproducción; la teoría de la diferenciación terminal, en que el envejecimiento celular se debe a una serie de modificaciones genéticas; la teoría de inestabilidad del genoma en las complejas interacciones entre el ADN, ARN y proteínas como causa del envejecimiento ${ }^{11}$; la teoría de mutación somática, en que la acumulación de las mutaciones en el ADN nuclear y la inestabilidad del genoma mitocondrial causarian las diferentes manifestaciones clínicas propias de enfermedades asociadas al envejecimiento ${ }^{12}$; es decir, establecen las bases de la aparición con la edad de trastornos cognitivos, alteraciones del movimiento, debilidad e incluso enfermedades como la diabetes, demencia y fallo cardíaco, siendo procesos que pueden postergarse o modularse para vivenciarlos con la mejor calidad de vida posible.

Asimismo, están: la teoría de los radicales libres, que provocarían efectos perjudiciales en los tejidos desencadenando patologías como las cardiovasculares, cáncer, cataratas, alzheimer y otras; la teoria error-catástrofe, en que los errores en la sintesis de proteínas causan la producción de proteínas anormales que provocan la senescencia ${ }^{13}$; la teoría de unidades cruzadas de estructuras celulares, que formarian enlaces moleculares entre cadenas de ácido nucleicos que aumentarian con la edad; la teoría inmunológica, que propone que el genoma nuclear programa cambios durante el desarrollo del organismo y su mismo deterioro revelaría la ancianidad $^{14}$; y, la teoría de acumulación de productos de desecho que es perjudicial para la reproducción y son rupturas de productos citoplasmáticos que llevan a la vejez. ${ }^{15}$

Otras teorias estiman que el envejecimiento es el resultado de una serie de procesos programados innatamente por el genoma, como la teoría de la capacidad de replicación finita de las células y varias otras teorías evolutivas, en que una de ellas señala que la vejez es una adaptación necesaria, programada como desarrollo, para el recambio y renovación de las poblaciones, donde los individuos más aptos son los que dejarían su impronta hereditaria a las generaciones siguientes; otra teoria postula que las mutaciones perjudiciales y su acumulación posteriormente causaría el envejecimiento $\mathrm{y}$, por último, otra teoría denominada evolutiva sugiere que la vejez es por un ajuste entre la supervivencia tardía y la fecundidad temprana. ${ }^{15}$
En consecuencia, todas estas teorías muy bien asentadas en la ciencia y desarrolladas a profundidad con un rigor indiscutible no reflejan una concepción multidimensional del envejecimiento, es decir, su orientación para unificar la vejez desde la dimensión biológica, social, psicológica y espiritual. Quizás finalmente considerando todas las teorías desde las distintas ciencias, es posible entender y situar el envejecimiento desde un contexto degenerativo más real.

El envejecimiento social es un proceso que sólo en la sociedad actual es visto como una transición desfavorable, y está influenciada su representación social por el lugar, la cultura y la condición socioeconómica. Se han establecido dos teorías al respecto, la primera denominada teoría de sistemas sociales que alude a construcciones mediáticas, que presenta la realidad propia de la vejez a través de códigos que orientan esta visión, la interpretan y dan ciertos atributos. Y segundo, la teoría de representaciones sociales que, tras la comunicación e interacción social, da respuesta a los estereotipos existentes en torno al envejecimiento. ${ }^{16}$ En ambas teorias sociales, el envejecimiento es reconstruido por la sociedad contemporánea a partir del lugar en que se vive y la idealización de la juventud. ${ }^{17,18}$

En relación al envejecimiento desde la dimensión espiritual, este tiene implicancia con la gratitud, el perdón y la alegría, un envejecer que espiritualmente se desarrolla en forma positiva y que la persona fortalece a medida que pasan los años al cuidar a otros, estar abierto a nuevas ideas, y mantenerse útil socialmente, al conservar la integridad en aceptar el pasado y pasar momentos en él para nutrirse de los logros presentes, junto con seguir dispuesto a aprender de la generación siguiente. Contribuyen también a una vejez espiritual sana el mantener fortalezas como la esperanza en la vida, esforzarse por ser todo lo autónomo posible, y valorar toda iniciativa, el disfrutar de la vida, mantener el sentido del humor y la capacidad para jugar, y ser tolerante con los aspectos desagradables de la vejez, cuidarse, aceptar las necesidades de dependencia y agradecer el apoyo recibido, y tratar de mantener contacto e intimidad con viejos amigos y lograr hacer nuevas amistades. ${ }^{19}$

Ciertamente el proceso de envejecer trae una serie de promotores genéticos que modulan lo biológico y psíquico, de caracteres sociales y comportamientos espirituales, que nos evidencia su perfil multidimensional, siendo innegable que los trabajadores de más edad suelen experimentar ciertos cambios de importancia en toda su integridad -cambios físicos y cognitivos con disminución visual y auditiva, aumento de índice de masa corporal, enfermedades cardiovasculares- en el propio trabajo por representar mayor riesgo de accidentes y enfermedades laborales. ${ }^{5}$ Además de estereotipos de poca capacidad laboral e incompatibilidad de esta con la vejez, y, en lo social, con la mirada del anciano como un ser pasivo que debiera estar exento de obligaciones y responsabilidades, limitado por la edad laboral de jubilación establecida socialmente. ${ }^{20}$

Para la mayor parte de la población, la vejez marca su comienzo con la jubilación, a pesar de que, legalmente, toda persona tiene el derecho a trabajar mientras posea las aptitudes necesarias, socialmente se considera al jubilado con un rol sin rol, más aun cuando se indignifica el descanso y se desvalida el ocio. ${ }^{20}$ Sin embargo, en la actualidad muchas personas en edad mayor se identifican aún con el trabajo asalariado y el sustento del hogar, sometidas a medidas de carácter pasivo y asistencial, con contratos de relevo y pocas iniciativas de formación. ${ }^{3,21}$ 
También se evidencian bajas contrataciones en personas adultas aunque sean igual de calificados que los más jóvenes ${ }^{4}$, y esto se basa en que la presión y estrés laboral pudiesen traer a la persona efectos negativos en la capacidad de trabajo, más aun si el trabajo trae consigo posiciones incómodas, cargas pesadas, largas horas en turno, ruidos, etc. que pudieran decantar en una futura jubilación por discapacidad. ${ }^{22}$

La salud juega un papel fundamental, pues las enfermedades promueven la jubilación anticipada por los sentimientos de incapacidad de funcionar laboralmente, de que el trabajo empeorará a futuro más su salud, y peor aun sentirse empujados por su empleador a jubilar por su mala salud, factores que finalmente limitarán la prolongación de los años de trabajo. ${ }^{23,24}$

El envejecimiento acelerado ha motivado el desarrollo de estudios que traten de mantener una buena salud, capacidad de trabajo, autonomía e integración social ${ }^{25}$, pues existen muchas creencias erróneas y mitos referente al desempeño de la persona mayor en el trabajo, como son ser menos capaz de aprender tareas nuevas, procedimientos o habilidades tecnológicas, incluso llevando a actitudes discriminatorias, actuando de barrera para adquirir empleo o evaluar el desempeño. ${ }^{4}$

Bajo estos acontecimientos, en el futuro la población laboral no requerirá de tantos trabajadores industrializados, más aun con el progreso tecnológico, sino que se trasladarán un mayor número al sector de servicios, el nivel de los trabajadores será con mejor formación y aumentará el número de mujeres, a pesar de su incorporación tardía al mercado. ${ }^{26}$ Por tanto, se hace perentorio concienciar sobre la contribución que las personas mayores pueden representar para el desarrollo socioeconómico y global de las sociedades. ${ }^{27}$

La vejez trae consigo una serie de consecuencias en las vidas de las personas que van más allá de una situación particular o de vivencia personal, sino que es todo un fenómeno de relevancia y consideración no sólo sanitaria, sino que también política, económica y social. La población envejecida requerirá una necesidad

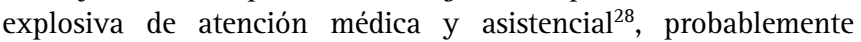
mucho de ese cuidado será trasladado además al Estado, aumentando el gasto público, previendo un peso económico importante para los países cuando esta población sea pasiva e improductiva, poniendo en manifiesto los recursos escasos y las necesidades ilimitadas ${ }^{20}$, amenazando las perspectivas de crecimiento económico, riesgo de financiamiento fiscal e inestabilidad macroeconómica. ${ }^{9,29}$ Pero también el perder estos empleados puede traer a las empresas pérdidas significativas en destrezas y conocimientos cruciales, donde la experiencia y la edad hacen a los trabajadores más eficaces, aumentando la productividad. ${ }^{30}$

La calidad de vida empeora a medida que se envejece, hay una mala respuesta social ante el deterioro biológico, probable pérdida de la ocupación, disminución del ingreso económico, presiones financieras, deterioro de la identidad social, ausencia de rol social, etc. $^{31,32}$ con un carácter segregado y poco atendido incluso por las instituciones públicas. ${ }^{26}$ Sumándose que a nivel de servicios sociosanitarios habrá mayor demanda, en el consumo de medicamentos, asistencia domiciliaria, hogares de residencia, atención prolongada, atención de especialidades de oncología, neurología, servicios de diagnóstico radiológico, tratamiento y rehabilitación por enfermedades como los accidentes cerebrovasculares, enfermedades degenerativas y crónicas. ${ }^{31,33-35}$

Debido a todas las implicancias que conlleva una población envejecida, se tornan relevantes las políticas económicas y sociales que se suministran a las generaciones más jóvenes en educación, trabajo, sistemas de pensión, salud e incorporación social para prever una generación anciana realmente protegida, siendo inclusive necesario la consideración de la adecuación de las viviendas, adaptación de los medios de trasporte, comercio, arquitectura de las ciudades, oferta alimenticia, entre otros aspectos, para disminuir los consecuencias negativas del envejecer ${ }^{31,36}$, pues la fuerza laboral de los países estará representada por las personas de mayor edad, donde la consideración del desarrollo humano como un fenómeno multidimensional logrará que la vejez sea vivida mejor y con calidad, protegiendo la salud funcional, disminuyendo los factores precursores de dependencia, fomentando el autocuidado, la identidad y autonomía del trabajador mayor, adentrándonos en la reflexión de: ¿qué hacer ante esta tendencia, cuando la fuerza laboral envejece?

En la actualidad el mercado laboral ha tenido varias trasformaciones que parecen dejar fuera a las personas mayores, ha aumentado el nivel de exigencias por medio de la incorporación de nuevas y sofisticadas tecnologías, los trabajadores se desarrollan en varios ámbitos y deben ser integrales para desempeñar cualquiera de los roles exigidos, hay movilidad de los trabajadores en una misma entidad, empleos nuevos y atípicos como el trabajo en domicilio o teletrabajo, que exigen capacidad de acción, alta producción, manejo de tiempos, trabajo en equipo y creatividad. El ser adulto mayor en actividad laboral implica desventajas y ventajas para los empleadores como lo haría cualquier otro trabajador indistintamente de la edad; estos tienen las facultades plenas para su desempeño y muchos de los factores determinantes en su potencial tienen que ver con las disposiciones de la misma empresa. Una empresa comprometida con el bienestar de sus trabajadores mayores tiene una organización funcional, en buenas y adaptables condiciones físico-ambientales que les propicien oportunidades y formación.

Las condiciones físico-ambientales del puesto de trabajo deben estar a la vanguardia de las características de la población envejecida, reduciendo esfuerzos físicos y redistribuyendo las tareas en relación a los principios ergonómicos, visualizando la adaptación del trabajo a las habilidades y competencias del trabajador, flexibilizando los espacios y los tiempos mientras se establezcan claramente los resultados que se esperan de su desempeño laboral, incluso generando planes de acción para implementar medidas básicas orientadas en la promoción de estilos de vida y ambientes de trabajo saludables.

Los ambientes de trabajo saludables deben contar con estamentos específicos destinados a la promoción y la prevención de la salud durante toda la vida laboral, y abocados a las condiciones de salud que enfrenta la sociedad a medida que envejece, además de las acciones destinados a la seguridad en el trabajo y la pesquisa de factores de riesgo de accidentes o enfermedad ocupacional.

Las intervenciones de promoción y prevención de la salud deben ir enfocadas en los estilos de vida saludable, promoviendo actividad física, alimentación equilibrada, ocio y entretenimiento, respeto de los tiempos con la familia, el trabajo y las relaciones sociales, previniendo la obesidad, el consumo de drogas y alcohol, el estrés laboral y las enfermedades ocupaciones, incluso motivando al personal a acudir y acceder periódicamente a sistemas de pesquisa de factores de riesgo cardiovascular, enfermedades crónicas, enfermedades de salud mental y disminuciones sensoriales.

En la protección de la salud mental de los trabajadores, las empresas deben reforzar sus sistemas de capacitación continua y transversal en toda la evolución y vida laboral, permitiendo que a cualquier edad el empleado esté calificado para incorporarse a 
cualquier rol o desafío, esté actualizado en conocimientos con posibilidades de perfeccionamiento e incentivando la puesta en práctica de los nuevos saberes. Y en el trabajo diario, establecer métodos de aprendizaje según las aptitudes y particularidades de cada trabajador, instaurando formadores con experiencia que apoyen a las nuevas generaciones, siempre evaluando la necesidad de capacitaciones o transferencia de conocimientos.

Las acciones que comprometen a las empresas exigen mejorar la redes en salud y la conexión de las organizaciones asistenciales con el mundo laboral, la empresa privada y trabajo informal, canalizando controles de salud repetidos a los trabajadores, brindándoles protección a todo nivel con una política intersectorial. Todas las estrategias deben apuntar a que nuestra sociedad reconozca el impacto que tiene la salud para que el adulto mantenga la independencia y autonomía necesarias para un buen desempeño laboral, propiciándose el envejecimiento activo, definido por la Organización Mundial de la Salud como "el proceso de optimización de las oportunidades en salud, participación y seguridad con el fin de mejorar la calidad de vida a medida que las personas envejecen" ${ }^{3}$

Para equiparar la demanda en salud que supone el envejecimiento de la fuerza laboral y la necesidad de prolongar sus años de trabajo, resguardando la calidad de vida en conjunto con la salud en las empresas, los gobiernos deben promulgar y fiscalizar políticas de trabajo decentes en ambientes saludables, regulando las condiciones mínimas requeridas para calificar los entornos laborales como promotores de la salud y protectores de riesgos y accidentes ocupacionales, estableciendo los requisitos de infraestructura concernientes a espacio por persona, ventilación, calefacción, iluminación, acceso a descansos, comodidad, confort y disposición de elementos de protección.

Las politicas de trabajo con ambientes saludables se respaldan con evidencia científica; por lo tanto, los gobiernos deben aumentar y potenciar el financiamiento de investigaciones que relacionen el ámbito de la salud y el trabajo, que propicien la generación de conocimientos en relación a la seguridad en el trabajo, la comprobación de intervenciones efectivas en la prevención de accidentes y promoción de la salud, y la vinculación efectiva de los estilos de vida saludables y los factores determinantes de la salud con los puestos de trabajo. Para ello se necesita personal sanitario calificado, debiendo también apoyarse económicamente su formación profesional con especialidades implicadas en la atención de la población envejecida y en grados académicos para propiciar la investigación.

Los gobiernos, a través de sus estamentos sanitarios, deben generar un acabado programa de atención al adulto que asegure el envejecimiento activo, con énfasis en la promoción y la prevención. Con preferencia en la población de trabajadores desde los 25 a los 60 años, con cobertura mejorada de servicios, diferenciados e integrados en la atención primaria en salud. Principalmente integrando las empresas en la satisfacción de necesidades de salud de las personas, todo por medio de la asistencia a controles periódicos y programados con elaboración de una rutina de pesquisa de riesgos cardiovasculares, enfermedades crónicas, cáncer y problemas osteomusculares, sumando además evaluación de riesgos laborales y de adquisición de enfermedades ocupacionales.

La Enfermería del trabajo y la Enfermería de atención primaria en salud juegan un rol trascendente para apoyar las distintas iniciativas para que la población viva más años saludablemente, cumpliendo con los siguientes estándares de "buen funcionamiento físico y mental, con un número reducido de trastornos crónicos, buena movilidad y capacidad para llevar una vida inde- pendiente, buena función cognitiva y ausencia de depresión"37, liderando y encausando la promoción y prevención en salud para un envejecimiento activo, saludable y con una buena calidad de vida, con hincapié en el fortalecimiento de los equipos interdisciplinarios en materias de atención del adulto trabajador.

Enfermería debe establecer una vinculación efectiva con las empresas para la realización de evaluaciones y exámenes destinados a evaluar capacidades y habilidades de los trabajadores para prevenir riesgos, fortalecer las debilidades o acomodar el puesto de trabajo. Para ello, debe diseñar, difundir e implementar guías clínicas y protocolos de atención del adulto con enfoque en promoción y prevención de enfermedades más prevalentes $\mathrm{y}$ ocupacionales. Y en su atención integral, también fortalecer la educación en autocuidado y adhesión a controles de salud, para poder establecer los factores protectores de la persona inmersa en su trabajo, con una vinculación reglamentada y apoyada gubernamentalmente, con el fin de dar al trabajador los recursos necesarios para que cuide su salud de manera responsable.

\section{CONCLUSIÓN}

El origen del envejecimiento está modelado por una serie de teorías que, en su conjunto, caracterizan al proceso como multidimensional. Las teorias biológicas, a pesar de su fundamentación genética, se configuran con el medio ambiente y explican algunas patologías más prevalentes en la vejez como los deterioros cognitivos. Las teorías del ámbito social hacen referencia a la representación mediática del adulto mayor jubilado con un rol pasivo en la sociedad, mientras que el envejecimiento desde la dimensión espiritual se vivencia sanamente como una etapa de entendimiento, plenitud, conformidad y positivismo ante el pasado y el presente.

El envejecimiento de la fuerza laboral es un fenómeno que actualmente vivenciamos en nuestra sociedad y la prolongación del período como trabajadores activos se ve limitado por la condición de salud. En la protección de la salud y la prevención de enfermedades prevalentes en la vejez y ocupacionales, el rol de promoción y prevención recae en diversas entidades, donde las empresas, los gobiernos, los sistemas de salud y Enfermería, en conjunto, deben propiciar lineamientos y oportunidades para un envejecimiento activo y saludable.

La tendencia de la fuerza laboral envejecida invita a las empresas a desarrollar estrategias para que los años laborales tengan calidad de vida y salud, adaptando el trabajo según las habilidades y competencias del trabajador con su situación general más allá de su edad, y otorgando puestos de trabajo saludables por medio de una entidad particular dedicada a este fin.

Los gobiernos deben diseñar e implementar nuevas políticas de salud laboral y de financiamiento de investigaciones, y formación profesional que aporten conocimientos a la relación de salud del trabajador envejecido y su puesto de trabajo. Además, a través de los sistemas de salud debe potenciar la atención y el cuidado de las generaciones más jóvenes pronosticando un sano envejecimiento, en donde los profesionales de enfermería juegan un rol preponderante en las acciones destinadas al fomento de estilos de vida saludables, la protección de la salud y la prevención de enfermedades cardiovasculares, problemas osteomusculares y alteraciones cognitivas, sumando además evaluación de riesgos laborales y de adquisición de enfermedades ocupacionales, a través de una atención integral y una vinculación intersectorialidad efectiva. 
1. Chackiel J. El envejecimiento de la población latinoamericana: ¿hacia una relación de dependencia favorable?. Santiago de Chile: CELADE-CEPAL; 2000. Población y Desarrollo; 4.

2. Gómez J, Saiach S, Lecuna N. Envejecimiento. Rev Fac Med UNAM. 1998;41(5):198-206.

3. Montero D, Fernández P. Calidad de vida, inclusión social y procesos de intervención. Bilbao: Universidad de Deusto; 2012.

4. Nazar G, Figueroa C. Creencias estereotípicas sobre el desempeño laboral de trabajadores mayores en Chile. Psicoperspectivas. 2015; 14(1):114-125.

5. Rodriguez A, Álvarez L. Repercusiones del envejecimiento de la población cubana en el sector salud. Rev Cubana Salud Pública. 2006; 32(2):178-82.

6. Lee D, Flemming L, Leblanc W, Arheart K, Ferrero K, Pitt-Catsouphes M, et al. Health Status and Risk Indicator Trends of the Aging U.S. Health care Work force. J Occup Environ Med. 2012; 54(4): 497-503.

7. Das M, N'Diaye P. El fin de la mano de obra barata. Finanzas \& Desarrollo. 2013;50(2):34-37.

8. Ross D. Ageing and work: an overview. Occup Med. 2010; 60:169-171.

9. Corone G, Costello D. ¿Llega Europa a la tercera edad? Finanzas \& Desarrollo. 2006; 9:28-31.

10. Sánchez M, Díaz P. Sobre envejecimiento activo e intergeneracionalidad. Reflexiones pensando en los profesionales sociales. Revista portal de Divulgação [en línea] 2012 [citado 12 may 2015]; 3(28): 20-27. Disponible en: http://www.portaldoenvelhecimento.org.br/revista/index.php.

11. Slagboom P, Vug J. Genetic inestability and aging: theorias, facts and future perspectives. Genome. 1989; 31:373-385.

12. Wallace $D$. Mitochondrial genetics: a paradigm for aging and degenerative diseases?. Science. 1992; 256: 628-632.

13. Toussaint 0 . Biología del envejecimiento celular. Convergencia de las teorias sobre el envejecimiento celular hacia el concepto de umbral crítico de acumulación de errores. Rev Gerontol. 1993; 3:143-152.

14. Brownleem M. Glycosylation products as toxic mediators of diabetic complications. Annu Rev Med. 1991; 42:159-166.

15. Pardo G. Consideraciones generales sobre algunas de las teorias de envejecimiento. Rev Cubana Invest Biomed. 2003: 22(1):58-67.

16. Parales $C$, Ruiz E. La construcción social del envejecimiento y de la vejez: un análisis discursivo en prensa escrita. Rev Lat Am Psicol. 2012; 34 (1-2):107-121.

17. Gergen $K$, Gergen M. The New Aging: Self Construction and Social Values. Draft copy for K. W. Schaie. Social structures and aging. New York: Springer; 2000.

18. Tapia C, Varela H, Barra L, Ubilla D, Iturra V, Collao C, Silva R. Valoración multidimensional del envejecimiento en la ciudad de Antofagasta. Rev Med Chile. 2010; 138: 444-451.

19. San Martín C. La espiritualidad en el proceso de envejecimiento del adulto mayor. Hologramática. 2008; 1(8):103-120.
20. Moragas R. Gerontología social. Envejecimiento y calidad de vida. $2^{2}$ ed. Barcelona: Herder; 2008.

21. Nava-Bolaños I, Ham-Chande R. Determinantes de la participación laboral de la población de 60 años o más en México. Pap poblac. 2014; 20(81): 59-87.

22. Härmä M. Adding more years to the work careers of an aging workforce what works? Scand J Work Env Hea. 2011; 37(6):451-453.

23. Maldonado $C$, Yáñez M. Una aproximación al estudio del empleo en la tercera edad. Cuadernos del CENDES. 2014; 31(81): 95-110.

24. De Wind A, Geuskens G, Reeuwijk K, Westerman M, Ybema J, Burdorf $A$, et al. Pathways through which health influences early retirement: a qualitative study. BMC Public Health. 2013; 13: 292.

25. Sampaio 0, Augusto V. Envelhecimento e trabalho: um desafio para a agenda da reabilitação Aging and work: a challenge for the rehabilitation Schedule. Rev Bras Fisioter. 2012; 16(2): 94-101.

26. Helene D. El mercado laboral en Canadá, trabajadores a partir de una cierta edad: abstenerse. Revista de Gerontología. 1993; 3(4): 260-264.

27. Bernardini D. 2012 año europeo del envejecimiento activo y la solidaridad intergeneracional. Una mirada multidimensional a un fenómeno global. Pliegos de Yuste. 2013; 15:85-95.

28. Belsky J. Psicología del Envejecimiento. Madrid: Thomson-Paraninfo; 2000.

29. Vial J. Desafíos de la transición demográfica en Chile. Santiago: Banco Central de Chile; 2013.

30. Strack R, Baier J, Fahlander A. Cómo gestionar el riesgo demográfico. Harvard Bus Rev. 2008; (2):1-11.

31. Sloane P, Zimmerman S, D'Souza M. What Will Long-Term Care Be Like in 2040?. N C Med J. 2014; 75(5):326-330.

32. Marin P. Tiempo nuevo para el adulto mayor. Enfoque multidisciplinario. $2^{\text {a }}$ ed. Santiago de Chile: Pontificia Universidad Católica; 1993.

33. Yang $W$, Williams J, Hogan $P$, Bruinooge $S$, Rodríguez $G$, Kosty $M$, et al. Projected Supply of and Demand for Oncologists and Radiation Oncologists Through 2025: An Aging, Better-Insured Population Will Result in Shortage. J Oncol Pract. 2014; 10(1):39-45.

34. Dall T, Storm M, Chakrabarti R, Drogan O, Keran C, Donofrio P, et al. Supply and demand analysis of the current and future US neurology workforce. Neurology. 2013; 81(30): 470-478.

35. Freeman W, Vatz K, Griggs R, Pedley T. The Workforce Task Force Report Clinical implications for neurology. Neurology. 2013; 81(30):479-486.

36. Pérez A, Leco F. Envejecimiento, estancamiento poblacional y perspectivas demográficas en extremadura. Geographicalia. 2011; 59(60):309-321.

37. Otero $A$, Zunzunegui $M$, Béland $F$, Rodríguez $A$, García de Yévenes $M$. Relaciones sociales y envejecimiento saludable. Madrid: Universidad Autónoma de Madrid; 2006. 